\title{
Prevenção de Dano Esofágico Durante Ablação de Fibrilação Atrial por Desvio Mecânico do Esôfago
}

\author{
Prevention of Esophageal Damage During Ablation of Atrial Fibrillation by the \\ Esophagus Mechanical Deviation
}

\begin{abstract}
Enrique Indalécio Pachón Mateos ${ }^{1,2,{ }^{*}}$, José Carlos Pachón Mateos ${ }^{1,2,3}$, Ricardo Carneiro Amarante ${ }^{1,2}$, Carlos Thiene Cunha Pachón², Tasso Júlio Lobo², Tomás Guillermo Santillana Peña², Juán Carlos Zerpa Acosta ${ }^{2}$, Juán Carlos Pachón Mateos ${ }^{1,2,3}$, Felipe Ortêncio ${ }^{2}$, Christian Higuti1,2
\end{abstract}

ORCID IDS

Pachón-M EO (D) https://orcid.org/0000-0001-7509-0731

Pachón-M JC (D) https://orcid.org/0000-0002-5111-488X

Amarante RC (D) https://orcid.org/0000-0002-7181-8198

Pachón CTC (iD) https://orcid.org/0000-0003-3943-4722

Lobo TJ (D) https://orcid.org/0000-0002-2801-1903

\section{RESUMO}

A fibrilação atrial é a arritmia de maior prevalência na população mundial. Apesar do uso de antiarrítmicos, é de difícil controle clínico, ocasionando sintomas e principalmente gerando risco de um evento tromboembólico. A partir de 1998, por meio da ablação por radiofrequência, o tratamento da fibrilação atrial mudou completamente, porém junto a essa importante evolução também iniciaram as complicações advindas dessa técnica de tratamento ablativo. Além das estenoses das veias pulmonares causadas pela ablação e posteriormente corrigidas com a mudança da técnica, surgiram as fístulas átrio-esofágicas, devido à aplicação de radiofrequência na parede posterior do átrio esquerdo. Esta parede está bem próxima (0,5 cm em média) do esôfago, facilitando a formação da fístula que leva à morte quase 100\% dos pacientes acometidos, apesar das diversas medidas de tratamento já desenvolvidas. Para evitar essa grave complicação, vários autores criaram técnicas para proteger o esôfago incluindo seu desvio mecânico para uma região oposta à da aplicação de radiofrequência, aproveitando a sua mobilidade e facilidade de abordagem. O desvio mecânico do esôfago tem se mostrado a forma mais simples, barata e eficiente de proteger esse órgão da lesão térmica da radiofrequência durante a ablação da fibrilação atrial.

PALAVRAS-CHAVE: Ablação por cateter; Fibrilação Atrial; Fístula atrioesofágica; Desvio mecânico do esôfago; Proteção térmica do esôfago.

\author{
Peña TGS (D) https://orcid.org/0000-0001-5795-9827 \\ Acosta JCZ (D) https://orcid.org/0000-0001-7436-2299 \\ Pachón-M J (D) https://orcid.org/0000-0003-4410-8753 \\ Ortencio F (D) https://orcid.org/0000-0003-1805-0420 \\ Higuti C (D) https://orcid.org/0000-0003-3820-8600
}

\begin{abstract}
Atrial fibrillation is the most prevalent arrhythmia in the world population. Despite the use of antiarrhythmics, it is difficult to control clinically, causing symptoms and mainly generating risk of a thromboembolic event. Since 1998, by means of radiofrequency ablation, the treatment of atrial fibrillation has completely changed, but together with this important evolution complications from this ablative treatment technique have also started. In addition to the pulmonary vein stenosis caused by the ablation and later corrected with the change in the technique, atrioesophageal fistulas appeared due to the application of radiofrequency in the posterior wall of the left atrium. This wall is very close $(0.5 \mathrm{~cm}$ on average) to the esophagus, which facilitates the formation of the fistula that leads to the death of almost 100\% of the affected patients, despite the various treatment measurements already developed. To avoid this serious complication, several authors have created techniques to protect the esophagus including its mechanical deviation to a region opposite to the radiofrequency application, taking advantage of its mobility and easiness of handling. The mechanical deviation of the esophagus has proven to be the simplest, cheapest and most efficient way to protect this organ from radiofrequency thermal damage during atrial fibrillation ablation.
\end{abstract}

KEYWORDS: Catheter ablation; Atrial fibrillation; Atrioesophageal fistula; Mechanical esophageal deviation; Thermal protection of the esophagus.

1.Universidade de São Paulo - São Paulo (SP), Brasil

2.Hospital do Coração - Serviço de Eletrofisiologia, Marcapasso e Arritmias - São Paulo (SP), Brasil

3.Instituto Dante Pazzanese de Cardiologia - Serviço de Marcapasso - São Paulo (SP), Brasil

Recebido: Jan. 28, 2020 | Aceito: Fev. 18, 2020

*Autor correspondente: epachon@usp.br

Editor de seção: José Tarciso Medeiros de Vasconcelos 


\section{INTRODUÇÃO}

O tratamento da fibrilação atrial (FA) por cateter foi descrito em 1998. Haïssaguerre ${ }^{1}$ descreveu nessa época, que os gatilhos da fibrilação atrial estavam localizados no interior das veias pulmonares (VPs), em bandas musculares oriundas das paredes atriais que penetravam nessas veias de forma desorganizada e aleatória. A aplicação de radiofrequência no interior das VPs resultou em elevada incidência de estenoses e recorrência da arritmia ${ }^{2}$.

Como consequência, a técnica foi modificada e o alvo da ablação por radiofrequência (RF) passou do interior dos óstios para o antro das $\mathrm{VPs}^{3}$. Além disso, com o intuito de aumentar o índice de sucesso, as linhas de bloqueio passaram a ser criadas pela RF na parede posterior do átrio esquerdo (AE) conectando as VPs. Essas linhas de bloqueio na parede posterior passaram a gerar um novo desafio, pois poderiam superaquecer o esôfago e causar lesões térmicas neste órgão.

A ablação da FA por cateter utilizando diferentes fontes de energia, tornou-se rotineira na maioria dos serviços de eletrofisiologia, devido ao melhor controle do ritmo sinusal quando comparado à terapia medicamentosa antiarrítmica ${ }^{4}$. O pilar do procedimento é o isolamento das $\mathrm{VPs}^{5}$. No entanto, para melhorar os resultados, vários desses serviços passaram a usar uma abordagem estendida para realizar ablações, principalmente nas formas persistentes da FA, implicando no mapeamento e na ablação de eletrogramas atriais fracionados (CFAEs) ${ }^{6}$, ninhos de $\mathrm{FA}^{7}$, na taquicardia de background (TBK) ${ }^{8,9}$, bem como na criação de linhas de bloqueio adicionais ${ }^{10}$, além do isolamento padrão das $\mathrm{VPs}^{5}$.

Adicionalmente à ablação por $\mathrm{RF}$, novas fontes de energia foram disponibilizadas (crioablação, laser e ultrassom) como também cateteres tecnicamente mais elaborados, os quais são capazes de produzir lesões mais profundas, criando o risco potencial de danificar estruturas próximas ao átrio esquerdo (AE), particularmente o esôfago ${ }^{11}$.

Uma das maiores preocupações e possíveis limitações durante o procedimento é o aquecimento do esôfago, que pode ocorrer quando a RF está sendo liberada na parede posterior do AE. Como consequência, são descritas erosões (esofagite) e úlceras, que variam de 2,9 a $47 \%{ }^{12}$ e fístula atrioesofágica (FAE), que é afortunadamente rara ${ }^{13}$.

A FAE tem sido descrita em pacientes submetidos a ablação cirúrgica de $\mathrm{FA}^{14} \mathrm{e}$ em pacientes submetidos à ablação de FA por cateter, usando radiofrequência ou crioablação ${ }^{15}$.
Apesar de a incidência da FAE ser de 0,1-0,25\% $\%^{16-18,}$ essa complicação aumenta a taxa de mortalidade em 80 a 100\%, sendo a segunda causa de morte mais frequente (16\%) relacionada ao procedimento de ablação da FA, ficando atrás apenas do tamponamento cardíaco agudo ${ }^{19,20 .}$ Os dados disponíveis na literatura sugerem que a ocorrência da FAE parece ser independente da experiência do operador ${ }^{21}$.

Embora várias estratégias estejam sendo desenvolvidas para evitar as lesões térmicas no esôfago, elas ainda ocorrem e têm sido relatadas na literatura ${ }^{13,22}$.

O objetivo deste artigo é revisar os aspectos fundamentais das lesões térmicas do esôfago, suas complicações, e quais técnicas estão sendo utilizadas atualmente para preveni-las.

\section{CORRELAÇÃO ANATÔMICA ENTRE ESÔFAGO E ÁTRIO ESQUERDO}

O esôfago médio é um tubo fibromuscular de $25 \mathrm{~cm}$ de comprimento e apenas $2 \mathrm{~cm}$ de largura. A posição do esôfago é frequentemente mais próxima dos óstios das VPs esquerdas em comparação aos óstios das VPs direitas ${ }^{23,24}$. Segundo Cury et al..$^{25}$, o diâmetro transversal médio do $\mathrm{AE}$ em contato com o esôfago é de 18,9 $\pm 4,4 \mathrm{~mm}$, enquanto que a distância média entre as duas estruturas é de $1,9 \pm 0,7$ mm (Fig. 1).

Com relação à inervação, o tronco vagal anterior passa externamente ao pericárdio, de 2,5 a 6,5 $\mathrm{mm}$ da parede posterior do átrio esquerdo ou das veias pulmonares ${ }^{26}$. Outro ponto muito importante é que tanto a irrigação como a inervação do esôfago ocorrem da porção externa (adventícia) para a porção mais interna da sua parede (mucosa). Dessa forma, qualquer lesão na porção externa da parede anterior tem um alto potencial de comprometer tanto a irrigação quanto a inervação esofágica, podendo determinar diversas consequências indesejadas.

\section{Fatores de risco}

Existem alguns fatores de risco mencionados na literatura que contribuem para a formação da fístula atrioesofágica, tais como: presença de úlcera esofágica; $\mathrm{AE}$ de maior diâmetro; baixo índice de massa corporal (IMC); aplicação prolongada de RF ou linhas sobrepostas na parede posterior do AE; utilização de bainhas deflectíveis, de cateteres de $8 \mathrm{~mm}$, de sondas ou tubos orogástricos ou nasogástricos; anestesia 


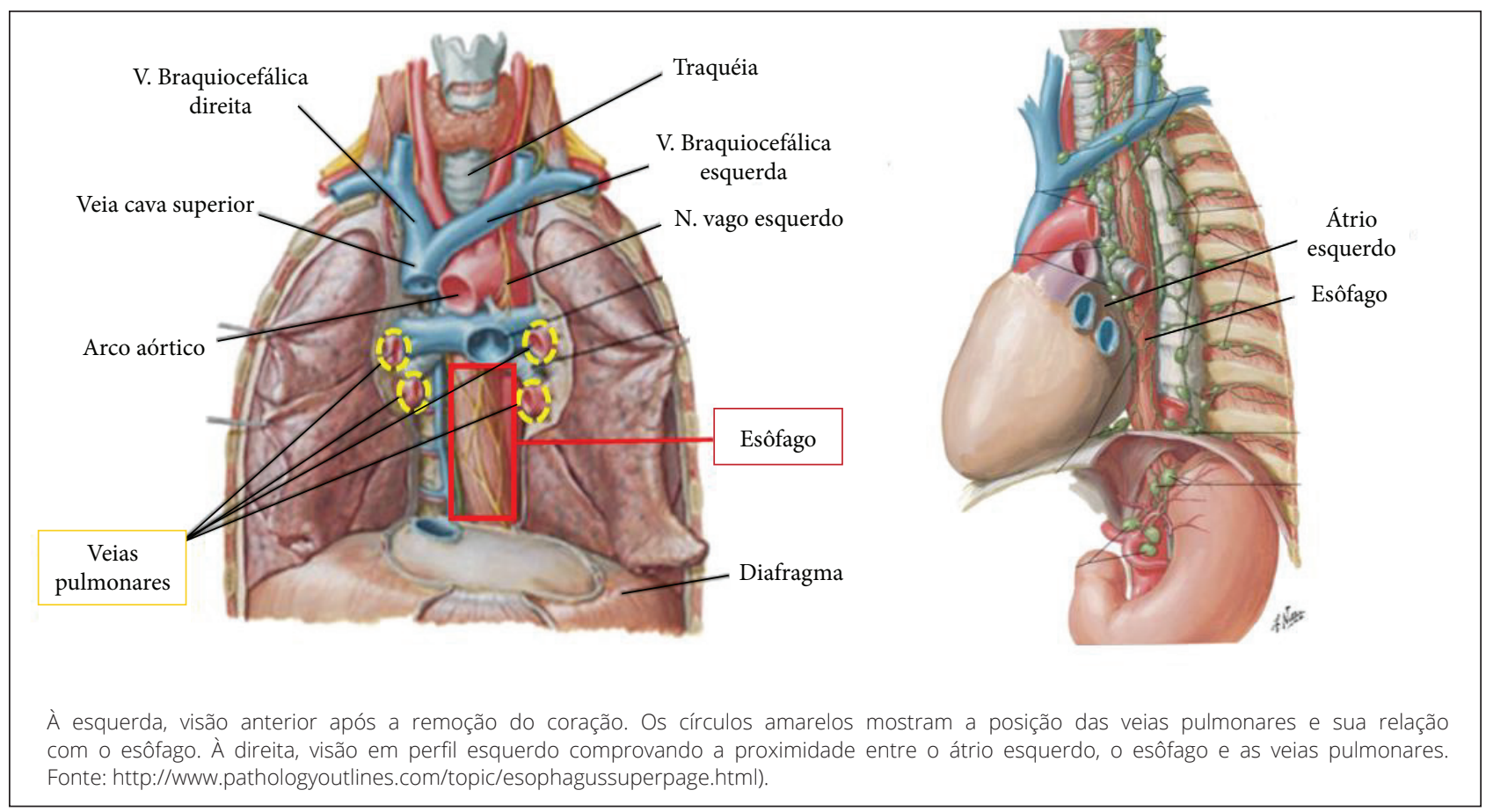

Figura 1. Relação anatômica entre o esôfago e o átrio esquerdo.

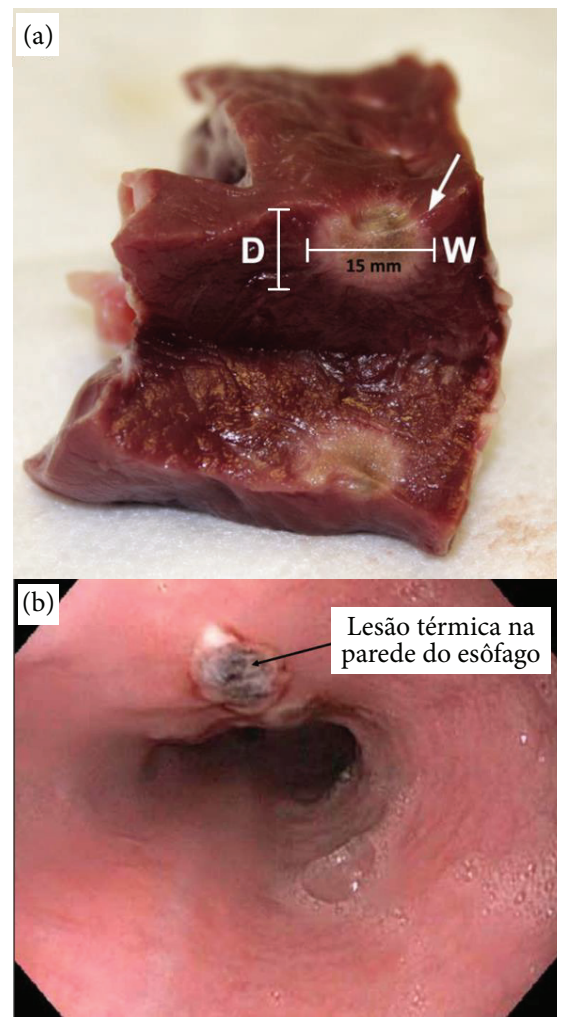

(a) Estudo experimental utilizando coração de porco. Observa-se que a lesão pode atingir profundidade de 10 a $15 \mathrm{~mm}$, suficiente para transpor a parede atrial e atingir o esôfago. (b) Caso da literatura mostrando uma lesão térmica da parede anterior do esôfago vista por endoscopia, a partir do lúmen da víscera, num paciente de 69 anos, queixando-se de disfagia um dia após ablação das veias pulmonares. Fonte: (a) Calzolari et al. ${ }^{29}$; (b) Chavez et al. ${ }^{30}$.

Figura 2. Profundidade da lesão térmica da ablação por radiofrequência. geral; potência máxima de $\mathrm{RF}(\mathrm{W})$ na parede posterior do $\mathrm{AE}$; e temperatura luminal esofágica elevada ${ }^{2,27,28}$ (Fig. 2). Há também maior incidência de lesão esofágica relatada com a navegação robótica quando comparada à ablação manual da FA usando parâmetros de energia de RF semelhantes ${ }^{18}$.

\section{MECANISMOS DE FORMAÇÃO DE FÍSTULAS ATRIOESOFÁGICAS}

De acordo com o entendimento atual, a FAE está relacionada ao comprometimento do suprimento sanguíneo arterial devido à oclusão das arteríolas terminais, infecção luminal esofágica e lesão do plexo vagal anterior, o qual compromete a motilidade do antro gástrico e do esfíncter inferior do esôfago e, como consequência, ocorre refluxo do conteúdo ácido gástrico 2,18,28.

Para Kapur et al. ${ }^{18}$, tanto a parede posterior do AE quanto a parede anterior do esôfago podem ficar comprometidas após a aplicação da RF. Em decorrência da falta de relatos de perfuração do AE na ausência de lesão esofágica, infere-se que a lesão primária (úlcera) ocorre no esôfago e progride em direção à parede posterior do $\mathrm{AE}$, determinando a formação da FAE.

O número reduzido de casos notificados de FAE desafiou a capacidade dos médicos de afirmarem quais fatores de 
risco são mais relevantes. No entanto Halbfass et al. ${ }^{31}$ demonstraram pela primeira vez, uma correlação direta entre a lesão térmica do esôfago, que ocorre após a ablação da FA, e a perfuração desse órgão. Os autores consideraram que a lesão térmica seria um pré-requisito para desencadear a série de eventos que determinam o desenvolvimento de FAE e que uma temperatura $>40,5^{\circ} \mathrm{C}$ no interior do esôfago está associada a um risco 2,1 vezes maior. Em contrapartida, até o momento não há um valor de variação de temperatura que garanta que não ocorrerá lesão térmica.

\section{APRESENTAÇÃO CLÍNICA}

Habitualmente, o diagnóstico da FAE ocorre entre 2 dias e 6 semanas após o procedimento de ablação. Han et al. ${ }^{32}$ descreveram que os pacientes com FAE geralmente apresentam febre como sintoma inicial em 73\% dos casos, além de sintomas neurológicos (déficits focais, convulsões, confusão mental e perda de consciência) em $72 \%$, sintomas gastrointestinais (hematêmese, melena, disfagia, odinofagia, náuseas e vômitos) em 41\% e sintomas cardíacos (dor torácica, dispneia e palpitações) em 40\%. Na sua análise multivariada, indicadores de risco de mortalidade foram: presença de sintomas neurológicos que apresentavam uma razão de probabilidade $(\mathrm{RP})$ de $16(\mathrm{p}<0,001)$ e presença de hemorragia digestiva, com RP de 4,22 ( $<<0,047$ ).

Outra complicação descrita é a gastroparesia, que é a obstrução gástrica devido à ausência de um componente mecânico ${ }^{33,34}$. Esses sintomas podem começar dentro de $3 \mathrm{~h}$ até 3 semanas após realização do procedimento de ablação. $\mathrm{O}$ mecanismo da lesão ocorre pelo dano dos plexos vagais que comprometem a motilidade e esvaziamento gástrico.

\section{DIAGNÓSTICO}

É muito importante diagnosticar com precisão as complicações do procedimento de ablação da FA, como a FAE e a gastroparesia. Em primeiro lugar, quando uma FAE está sendo considerada como um diagnóstico possível, recomenda-se realizar um hemograma com contagem de células brancas porque é um marcador laboratorial precoce e sensível dessa complicação. As hemoculturas de pacientes com sepse secundária à FAE geralmente desenvolvem organismos gram-positivos ${ }^{18}$. A confirmação do diagnóstico geralmente é feita por uma tomografia computadorizada (TC) ou uma ressonância magnética (RM) com contraste intravenoso (Fig. 3).

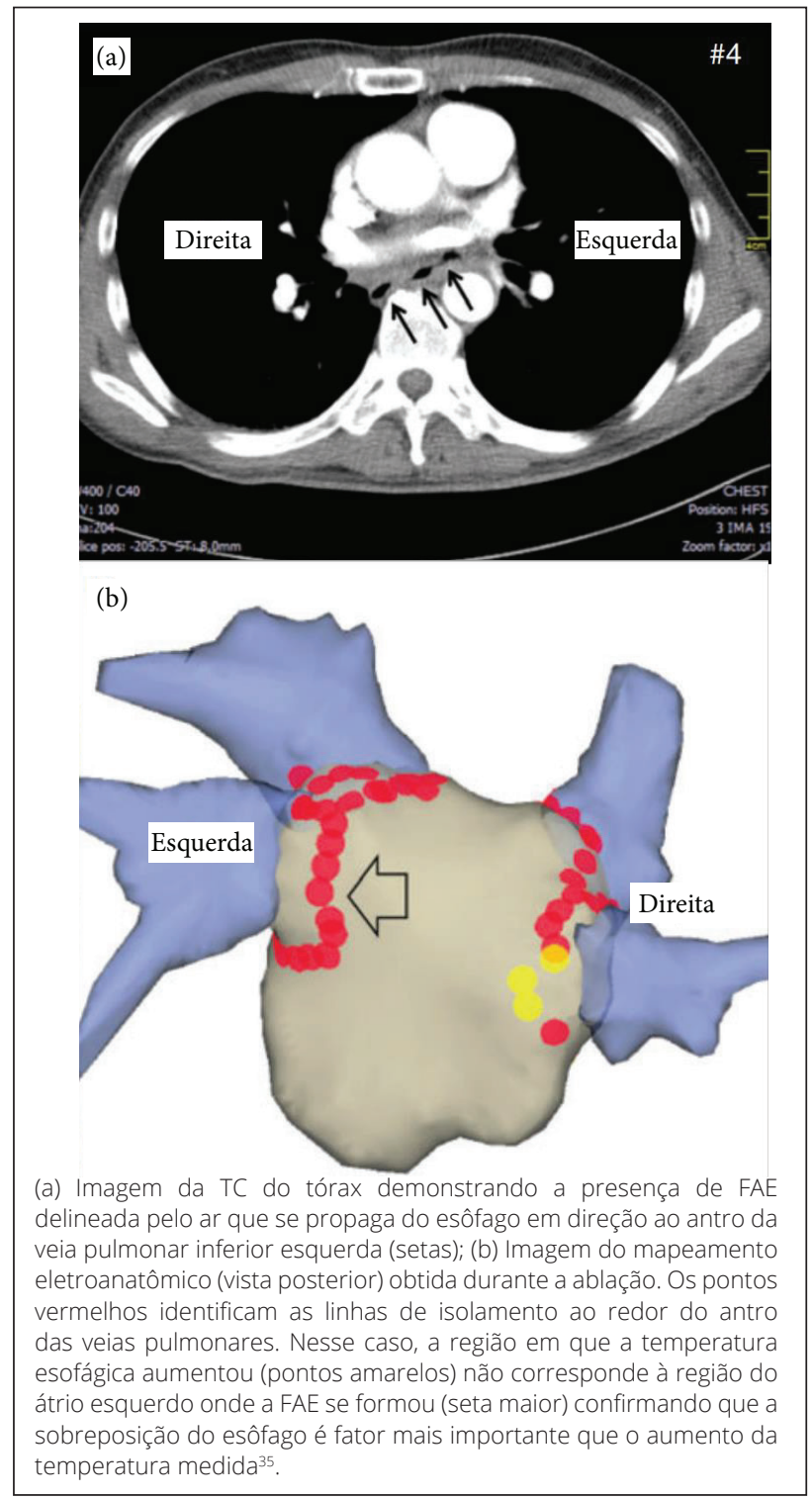

Figura 3. Relação entre as aplicações de RF e a formação da FAE.

Em ambos os casos, a fase inicial do exame pode indicar a presença de um pneumomediastino ou pneumopericárdio, enquanto a fase de contraste também pode indicar extravasamento de contraste entre o $\mathrm{AE}$ e o mediastino ou entre o AE e o esôfago. O uso do contraste oral é recomendado para complementar um possível diagnóstico de ruptura isolada do esôfago ${ }^{18}$.

É importante ter em mente que procedimentos como a endoscopia digestiva alta (EDA), que manipulam o esôfago, devem ser evitados, para prevenir que o risco de embolização aérea macica ocorra, devido a insuflação do esôfago. Em 
segundo lugar, se a suspeita for de gastroparesia, quando houver sintomas de atraso no esvaziamento gástrico, recomenda-se a realização de métodos que confirmem esse atraso, como cintilografia ou ressonância magnética ${ }^{49}$.

\section{TRATAMENTO}

Devido à baixa incidência de FAE, existem poucos estudos que possuem dados estatísticos significativos que apoiam uma terapia como a mais eficaz. Mesmo assim, durante a prática clínica, inibidores de bomba de prótons, inibidores de $\mathrm{H} 2$ e sucralfato são amplamente utilizados. São prescritos alguns dias antes do procedimento de ablação até 4 a 6 semanas após o procedimento, principalmente nos casos em que a lesão da mucosa esofágica é identificada ${ }^{36,37}$.

Uma vez estabelecido o diagnóstico, o tratamento deve ser realizado precocemente. Existem duas técnicas que podem ser utilizadas, a correção com a utilização de stent por via endoscópica ou a correção cirúrgica, que é a preferível, pois reduz significativamente a taxa de mortalidade ${ }^{13,20}$.

Em uma análise retrospectiva com 29 pacientes que apresentam FAE após o procedimento de ablação de FA, foi demonstrado que $100 \%$ dos pacientes que foram tratados com stent foram a óbito, ao passo que $59 \%$ dos pacientes submetidos ao procedimento cirúrgico sobreviveram ${ }^{38}$.

No caso de um diagnóstico de gastroparesia, dependendo do grau da lesão, o tratamento pode ser realizado através de modificação da dieta e suporte nutricional, agentes procinéticos, injeção intrapilórica de toxina botulínica e, ainda, utilizando estimulação elétrica gástrica. Nesses casos, os resultados positivos foram relatados dentro de semanas ${ }^{38}$.

Os pacientes devem ser bem orientados sobre a ampla gama de sintomas relacionados à lesão esofágica, antes da alta hospitalar, a fim de antecipar possíveis diagnósticos de complicações.

\section{TÉCNICAS PARA EVITAR O SUPERAQUECIMENTO DO ESÔFAGO}

Várias técnicas foram desenvolvidas para proporcionar maior segurança durante a aplicação de $\mathrm{RF}$ na parede posterior do $\mathrm{AE}$. Qualquer técnica usada com o objetivo de limitar ou impedir a liberação de $\mathrm{RF}$ em áreas próximas ao esôfago depende de informações precisas da localização específica desse órgão,em relação às áreas-alvo da ablação. Um dos maiores desafios é a ampla variação anatômica da largura e sua constante mobilidade.

A técnica ideal para evitar lesões térmicas esofágicas seria não aplicar nenhuma $\mathrm{RF}$ em regiões próximas ao esôfago. No entanto, muitas vezes há sobreposição entre a parede posterior do AE, VPs e o esôfago ${ }^{23}$. Essa abordagem seria menos eficaz porque não permitiria que toda a energia de $\mathrm{RF}$ necessária fosse aplicada nas regiões sobrepostas, o que poderia comprometer o resultado do procedimento ablativo. As imagens do esôfago obtidas antes da ablação da FA não são confiáveis para esse propósito, pois a mobilidade natural desse órgão pode alterar seu posicionamento a qualquer momento, ou seja, essas imagens deveriam ser obtidas em tempo real, durante a ablação.

Stárek et al. ${ }^{39}$ demonstraram que apenas em $35 \%$ dos casos houve uma correspondência entre a posição do esôfago antes e logo após o início do procedimento de ablação. Daoud et al..$^{40}$ também observaram uma modificação de até $50 \%$ no diâmetro do esôfago em $44 \%$ dos pacientes estudados que foram submetidos ao procedimento de ablação de FA. Sob sedação profunda e contraste de bário administrado por via oral, Good et al. ${ }^{41}$ obtiveram resultados semelhantes: $67 \%$ de seus pacientes apresentaram deslocamento lateral espontâneo do esôfago $\geq 2 \mathrm{~cm} \mathrm{e} 4 \%$ tiveram deslocamento $\geq 4 \mathrm{~cm}$.

Um dos métodos utilizados para a visualização esofágica durante a fluoroscopia, consiste na administração de contraste baritado via oral ou por sonda esofágica $(20-30 \mathrm{ml})$, o qual permanece coletado no seu interior por 30-60 $\mathrm{min}^{39}$. Dessa forma, usando a imagem radiológica, é possível visualizar a posição do esôfago em relação ao $\mathrm{AE}$ em tempo real. A principal limitação dessa técnica é que ela não permite delimitar as bordas esofágicas, pois apenas o seu lúmen é visualizado. No entanto, Stárek et al..$^{39}$ demonstraram que não houve diferenças significativas entre os diâmetros esofágicos quando estes foram avaliados por angiografia tridimensional e tomografia computadorizada.

Apesar dessa técnica permitir a visualização esofágica em tempo real, ela não é capaz de prevenir lesões térmicas com eficácia caso as aplicações de RF sejam efetuadas sobre esse órgão ${ }^{28}$.

\section{Ultrassom Intracardíaco}

A ecocardiografia intracardíaca (EIC), por outro lado, permite a visualização da posição do esôfago em relação ao $\mathrm{AE}$ em tempo real. A delimitação de suas bordas com a integração de suas imagens com o sistema de mapeamento eletroanatômico 3D também é possível e muito útil. Além 
disso, tem a vantagem de não usar a fluoroscopia para delimitar o esôfago, permite avaliar as regiões do esôfago que estão em contato com a parede posterior do $\mathrm{AE}$, além de observar a distância entre essas duas estruturas e o padrão de formação de microbolhas, as quais podem indicar o superaquecimento da parede atrial ${ }^{42}$. Mesmo assim, o EIC não permite modificar a posição do esôfago, caso haja sobreposição de uma áreaalvo para a ablação.

\section{Aparelhos radiopacos para delinear o esôfago}

Essa técnica pode ser realizada através de um termômetro esofágico ou com cateteres de eletrofisiologia que podem delinear as bordas luminais do esôfago e seu trajeto de maneira aproximada. Também pode-se posicionar um cateter no lúmen esofágico para que seja reconhecido pelo sistema de mapeamento eletroanatômico 3D e, assim, mostrar virtualmente as bordas do esôfago delimitadas por pontos ${ }^{43}$.

\section{Mapeamento eletroanatômico combinado com tomografia computadorizada ou ressonância magnética}

O uso de tomografia computadorizada ou ressonância magnética antes de um procedimento permite gerar imagens do AE e do esôfago. Essas imagens podem ser fundidas com a anatomia construída pelo sistema de mapeamento eletroanatômico 3D. Esse método tem uma restrição já mencionada anteriormente, pois ele gera imagens estáticas ${ }^{39,44}$. Com isso, modificações na posição do esôfago durante o procedimento podem ocorrer e não serem identificadas no momento da aplicação de RF na parede posterior do AE. Consequentemente, uma falsa sensação de segurança pode ser produzida durante a aplicação de RF.

\section{Titulação de potência RF (W)}

A técnica mais utilizada nos diversos serviços de eletrofisiologia é a titulação de potência aplicada na parede posterior do AE. Alguns autores recomendam a titulação da potência de $\mathrm{RF}$ em $\leq 25 \mathrm{~W}$ e ajustar a duração da aplicação de $\mathrm{RF}$ em $\leq 30 \mathrm{~s}^{28,37}$. De acordo com Bahnson et al. ${ }^{43}$ seria aconselhável limitar a potência de RF a valores abaixo de $20 \mathrm{~W}$ por $15 \mathrm{a} 20 \mathrm{~s}$ em cada aplicação, quando estiver próximo ao esôfago, e depois aguardar 180 s pelo menos, para que haja um resfriamento adequado do órgão. No entanto, mais dados são necessários para suportar essa abordagem.

Os maiores desafios dessa técnica permanecem em saber qual é a localização precisa das bordas do esôfago, bem como a quantidade segura de energia que seja suficiente para gerar uma lesão atrial transmural sem comprometer o esôfago ${ }^{43}$. Portanto, a titulação de potência de RF geralmente depende do uso de um termômetro esofágico que pode auxiliar no controle da quantidade de potência aplicada nessas regiões próximas ao esôfago.

\section{Monitoramento da temperatura esofágica luminal}

O monitoramento contínuo da temperatura do esôfago é capaz de detectar e fornecer alertas quando há um aumento na temperatura luminal durante a aplicação de RF. Na literatura, não há um limite preciso em relação à temperatura máxima para que a aplicação de RF possa ser interrompida devido ao risco de lesão esofágica. Vários pesquisadores já lidaram com esse problema. Alguns autores sugerem valores máximos entre 39 a $41{ }^{\circ} \mathrm{C}$, no entanto, a incidência de lesões esofágicas varia de 10 a $47 \%$ utilizando esses valores de corte ${ }^{12,45-47}$. Singh et al. ${ }^{49}$ demonstraram menor incidência de lesão esofágica em comparação com a não utilização de termômetro ( $6 \%$ vs $36 \%$; valor de $\mathrm{p}<0,006$ ), quando utilizado um ponto de corte de $38,5^{\circ} \mathrm{C}$ para interromper a aplicação de RF. $\mathrm{O}$ aumento $\geq 1{ }^{\circ} \mathrm{C}$ em relação à temperatura basal do esôfago pode ser utilizado como critério de corte $^{37}$, no entanto há relato de ocorrência de lesões esofágicas mesmo com menor aquecimento ${ }^{52}$. Além disso, a cada elevação de $1{ }^{\circ} \mathrm{C}$ em relação à temperatura basal esofágica, existe um OR de 1,36 para o desenvolvimento de lesão térmica ${ }^{48}$.

Müller et al. ${ }^{49}$ avaliaram um grupo de 80 pacientes por meio de endoscopia digestiva alta (EDA) dois dias após a ablação de FA. Os autores levantaram evidências de que o grupo em que o termômetro esofágico foi utilizado apresentava maior número de lesões no esôfago quando comparado ao grupo que não o utilizava ( $30 \%$ vs. $2,5 \%$, $\mathrm{p}<0,01)$, e, na regressão logística multivariada, o único preditor de risco independente para a formação de lesões esofágicas foi a presença do termômetro (RP 16,7, p < 0,01).

Em uma publicação posterior, Halbfass et $\mathrm{al} . .^{50}$, avaliaram o risco de usar um termômetro esofágico, agora com isolamento elétrico em seus polos de temperatura. A incidência de lesões esofágicas foi semelhante no grupo com e sem o dispositivo, 
respectivamente $(7,5 \%$ vs. $10 \%, \mathrm{p}=1,0)$. Dessa forma, os autores concluíram que a utilização de termômetros isolados eletricamente não está relacionada ao aumento do risco de lesões esofágicas. No entanto, embora haja redução, ainda são descritas lesões térmicas que demonstram que essa técnica de proteção esofágica não é suficiente para preveni-las.

\section{Refrigeração contínua do esôfago}

Uma forma de impedir o aquecimento do esôfago pode ser por meio de sistemas com balão intraesofágico ou instilando água fria intraluminal. Entretanto, apesar do resfriamento adequado da mucosa, um resfriamento suficiente das porções externas da parede esofágica não é garantido ${ }^{52,53}$. Ocorre também um deslocamento do esôfago em direção à parede posterior do $\mathrm{AE}$ após a insuflação do balão, o que, teoricamente, pode aumentar a risco de lesão térmica.

\section{Desvio mecânico}

Considerando que o esôfago está anatomicamente muito próximo da parede posterior do $\mathrm{AE}$, tem o trajeto variável e apresenta mobilidade no mediastino posterior, surgiu a possibilidade de mover esse órgão de regiões com risco de aquecimento utilizando dispositivos inseridos no lúmen esofágico.

Essa técnica idealizada por Pachón et al. ${ }^{53}$ constitui o desvio mecânico contralateral do esôfago através da utilização do próprio transdutor do ecocardiograma transesofágico (T-ETE) (Fig. 4). De acordo com a região em que a RF é aplicada no $\mathrm{AE}$, o esôfago é deslocado lateralmente para a posição oposta, por meio da manipulação do T-ETE, obtendo-se, na grande maioria dos casos, uma distância suficiente para evitar o aquecimento desse órgão. Além disso, um termômetro multicanal pode ser inserido no lúmen esofágico para monitorar a temperatura durante a aplicação de $\mathrm{RF}^{55}$. Caso alguma elevação de temperatura seja constatada, o desvio do esôfago é reavaliado e otimizado para permitir a aplicação de forma segura e completa. De acordo com esses trabalhos, nenhum caso de fístula atrioesofágica ou complicação importante relacionada ao esôfago foi observado, tendo essa técnica sido usada em um grande número de casos (704 pacientes) ${ }^{54}$ (Fig. 5).

Mais tarde, Chugh et al..$^{55}$ também descreveram o uso da técnica, porém realizaram o desvio mecânico do esôfago com o T-ETE e esse dispositivo foi posteriormente removido com a justificativa de possível efeito antena (efeito por indução entre transdutor e cateter de RF). Nessa condição, o esôfago retornou à sua posição basal em 7 dos 9 casos, sendo pouco eficaz.

Mais recentemente, em 2012, Koruth et al. ${ }^{56}$ realizaram o desvio mecânico em 20 pacientes, utilizando um guia pré-moldado que foi inserido por uma sonda nasogástrica. Assim, o esôfago poderia ser deslocado para regiões mais seguras, impedindo ou reduzindo seu aquecimento. Segundo a investigação dos autores, o desvio médio foi de $2,8 \pm 1,6 \mathrm{~cm}$ para a esquerda e $2,8 \pm 1,8 \mathrm{~cm}$ para a direita. Os autores descreveram que a temperatura esofágica atingiu valores $\geq 38,5^{\circ} \mathrm{C}$ em $3 / 20$ casos e em nenhum paciente houve registro acima de $40^{\circ} \mathrm{C}$. Dos 20 pacientes incluídos,

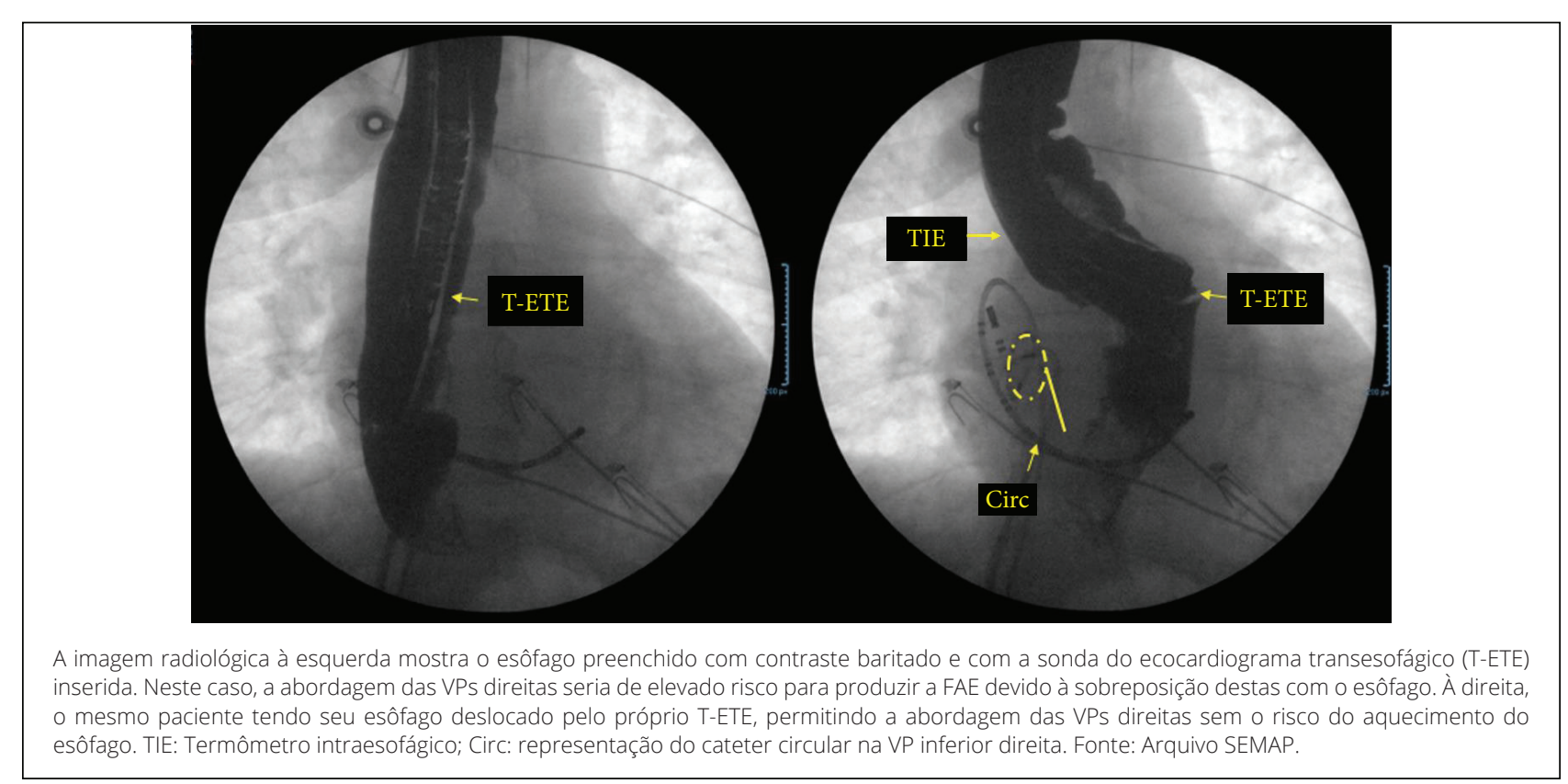

Figura 4. Técnica idealizada por Pachón et al. ${ }^{53}$ para proteção do esôfago durante a ablação da fibrilação atrial. 
63\% apresentavam lesões esofágicas superficiais relacionadas ao trauma, mas sem repercussões clínicas.

Outros fabricantes desenvolveram sistemas capazes de afastar o esôfago mecanicamente da sua posição original para uma posição distante do local de aplicação de RF na parede posterior do AE (Fig. 6). Destacam-se o Esosure (Northeast Scientific Inc.) que é simplificadamente um fio metálico de nitinol com duas curvas pré-formadas. Essas curvas são suaves em temperatura ambiente facilitando a sua introdução, porém, ao aquecer com a temperatura corporal, ficam bem proeminentes promovendo o deslocamento do esôfago. A movimentação do dispositivo permite escolher a posição mais adequada de acordo com o local onde a RF será aplicada.

Outro dispositivo criado para o deslocamento do esôfago é denominado DV8 (Manual Surgical Sciences). Ele permanece retificado em condições basais permitindo sua inserção pela cavidade oral. Posteriormente um balão interno é insuflado, modificando o formato e permitindo o desvio do esôfago. Para facilitar sua visualização, uma via permite preenchê-lo com contraste orientando o nível e o grau de deslocamento desejado.

\section{DISCUSSÃO}

A proximidade da parede posterior do $\mathrm{AE}$ em relação ao esôfago é extremamente relevante no cenário da ablação da FA, e não é apenas uma consideração anatômica. A abordagem antral e principalmente circunferencial das VPs resulta em maior convergência das linhas de bloqueio na parede posterior do AE. Também é possível que as taquicardias residuais apareçam após a ablação da FA, na parede posterior do AE, do tipo flutter atípico e TBK.

Assumindo que a lesão térmica direta do esôfago é o provável mecanismo fisiopatológico da FAE, é necessário o desenvolvimento de técnicas que permitam a aplicação da potência e duração da RF necessárias para o tratamento
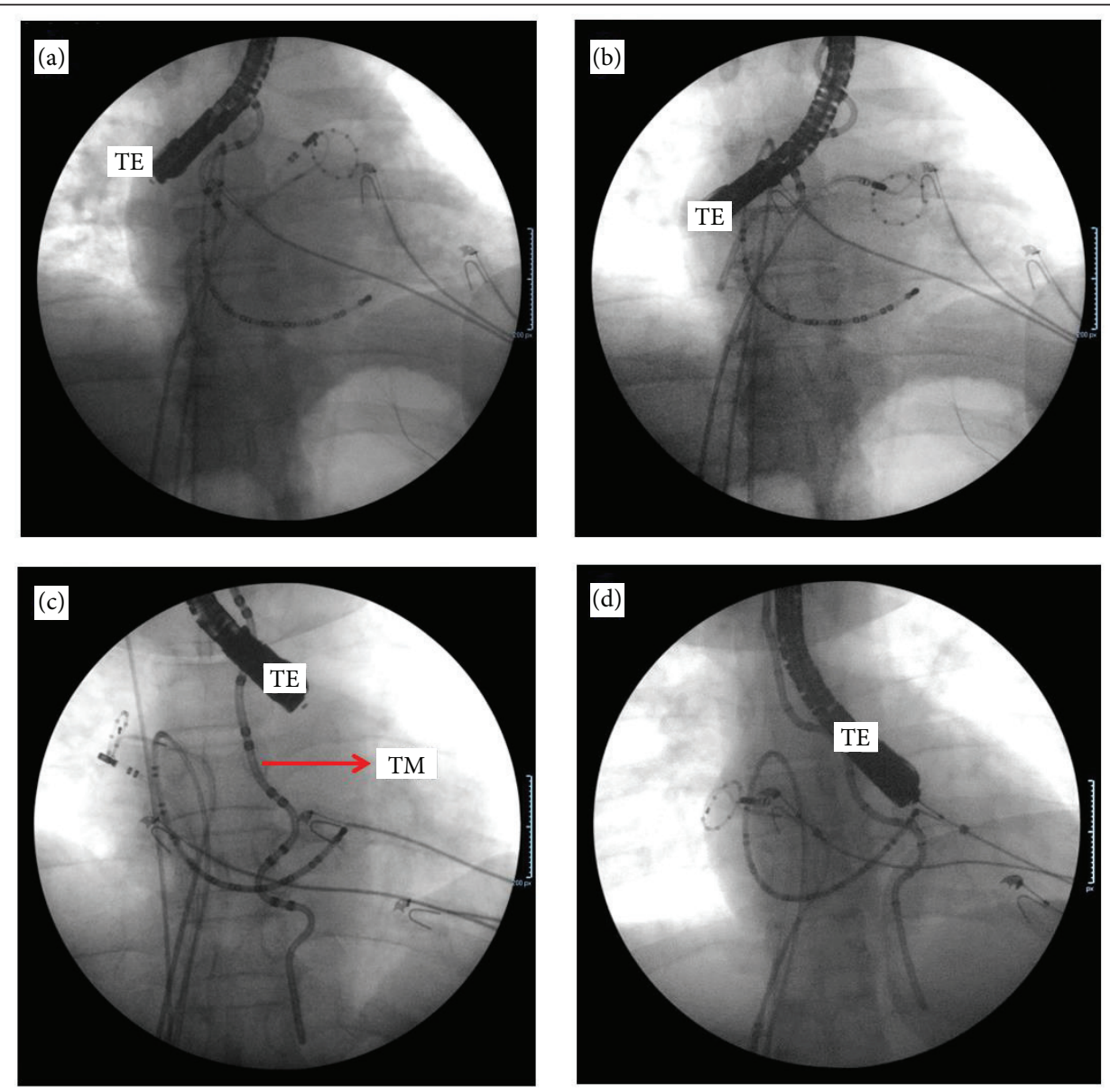

Utilizando o T-ETE é possível afastar o esôfago para abordar todas as VPs (a, b, c, d) sem risco do aquecimento esofágico. Para comprovar a segurança, um termômetro multicanal (TM) (Circa) também é posicionado na luz do esôfago permitindo confirmar que houve deslocamento completo do órgão, e não apenas da sua parede lateral, além do monitoramento contínuo da temperatura. Fonte: Arquivo SEMAP.

Figura 5. Imagens radiológicas obtidas durante procedimento de ablação de FA conforme a técnica de Pachón et al ${ }^{53}$. 


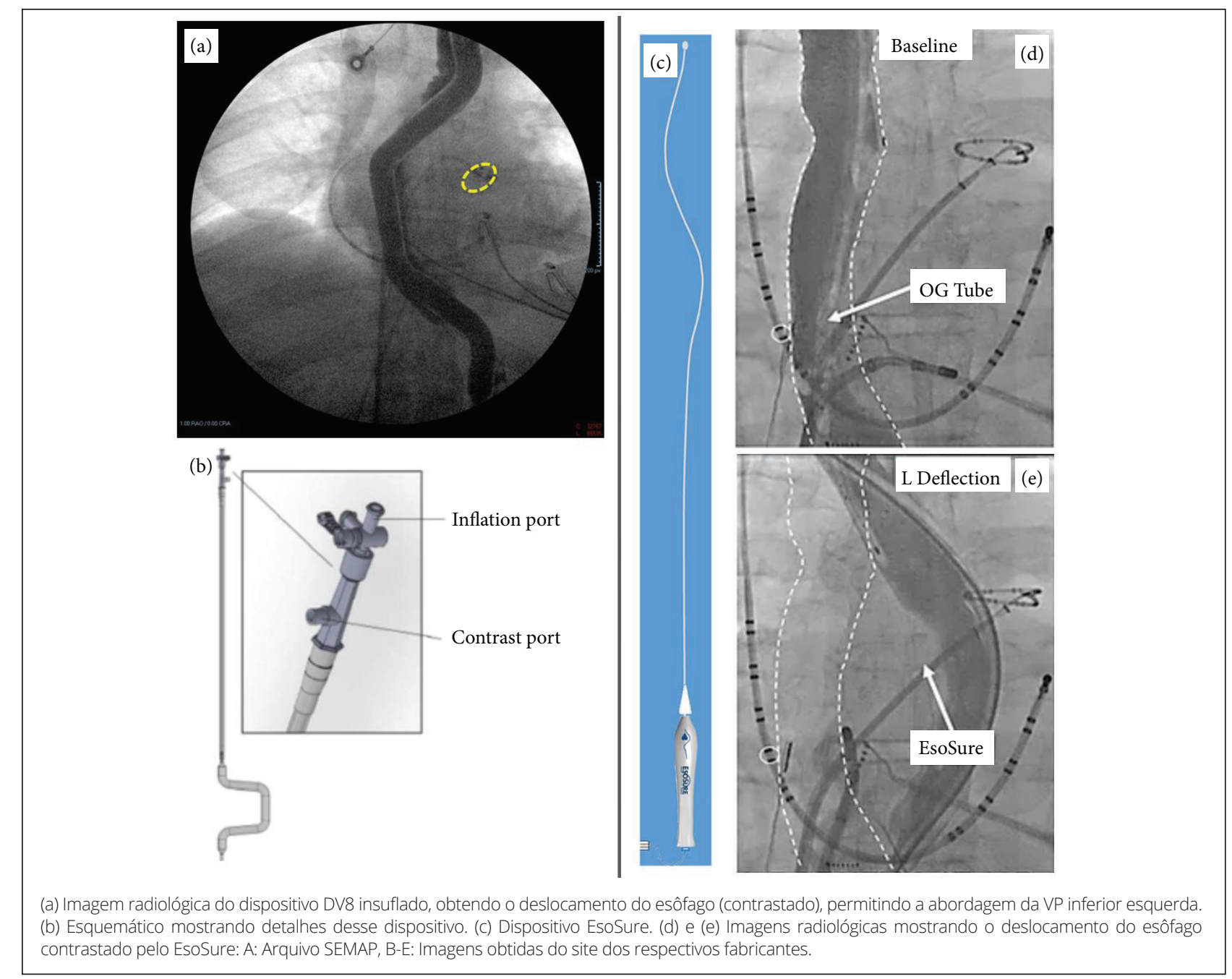

Figura 6. Imagens mostrando dois dispositivos disponíveis comercialmente para obter o deslocamento do esôfago durante a ablação da FA.

adequado dessas regiões críticas e que, ao mesmo tempo, evitem aquecer o esôfago. Embora existam várias estratégias disponíveis para evitar o sobreaquecimento do esôfago, identificando a sua localização; reduzindo a quantidade ou a duração da energia de RF fornecida; por monitorização da temperatura do esôfago ou por refrigeração contínua do lúmen do esôfago. Ainda assim é possível observar seu sobreaquecimento, e nenhuma delas modifica a posição do esôfago, a fim de evitar lesão pelo aumento da temperatura.

No monitoramento da temperatura por termômetro intraesofágico, apesar de bastante útil, deve-se considerar que é a temperatura da mucosa esofágica, a porção mais interna do órgão, que está sendo medida. Quando essa temperatura se eleva, as porções externas do esôfago já atingiram valores bem superiores, comprometendo justamente as áreas mais sensíveis, compostas pela irrigação e a inervação. É conveniente reduzir drasticamente os níveis de temperatura detectados por esse sistema para evitar que as regiões externas atinjam níveis de risco. $\mathrm{Na}$ técnica desenvolvida por Pachón et al. ${ }^{53}$, o termômetro intraesofágico emite um alerta quando a temperatura local ultrapassa apenas $0,5^{\circ} \mathrm{C}$ da temperatura corporal e um potente alarme caso atinja $1^{\circ} \mathrm{C}$ acima desse valor (Fig. 7) ${ }^{54,55}$. Com tais parâmetros, as camadas externas do esôfago são submetidas a pequenas variações de temperatura, dentro da faixa fisiológica, não deletérias para o mesmo. Outro dado importante a ser considerado é que os sensores do termômetro são perifericamente posicionados em uma sonda com formato senoidal, de tal forma que eles ficam apoiados nas porções laterais do esôfago, locais mais próximos do antro das VPs, que são os pontos mais vulneráveis durante a ablação da FA.

Até agora, o deslocamento mecânico do esôfago parece ser a abordagem mais eficaz e mais simples, pois permite aplicar toda a RF necessária na parede posterior do AE, sem limitação da potência, da temperatura ou do tempo. $\mathrm{O}$ uso de anestesia geral para realizar o 


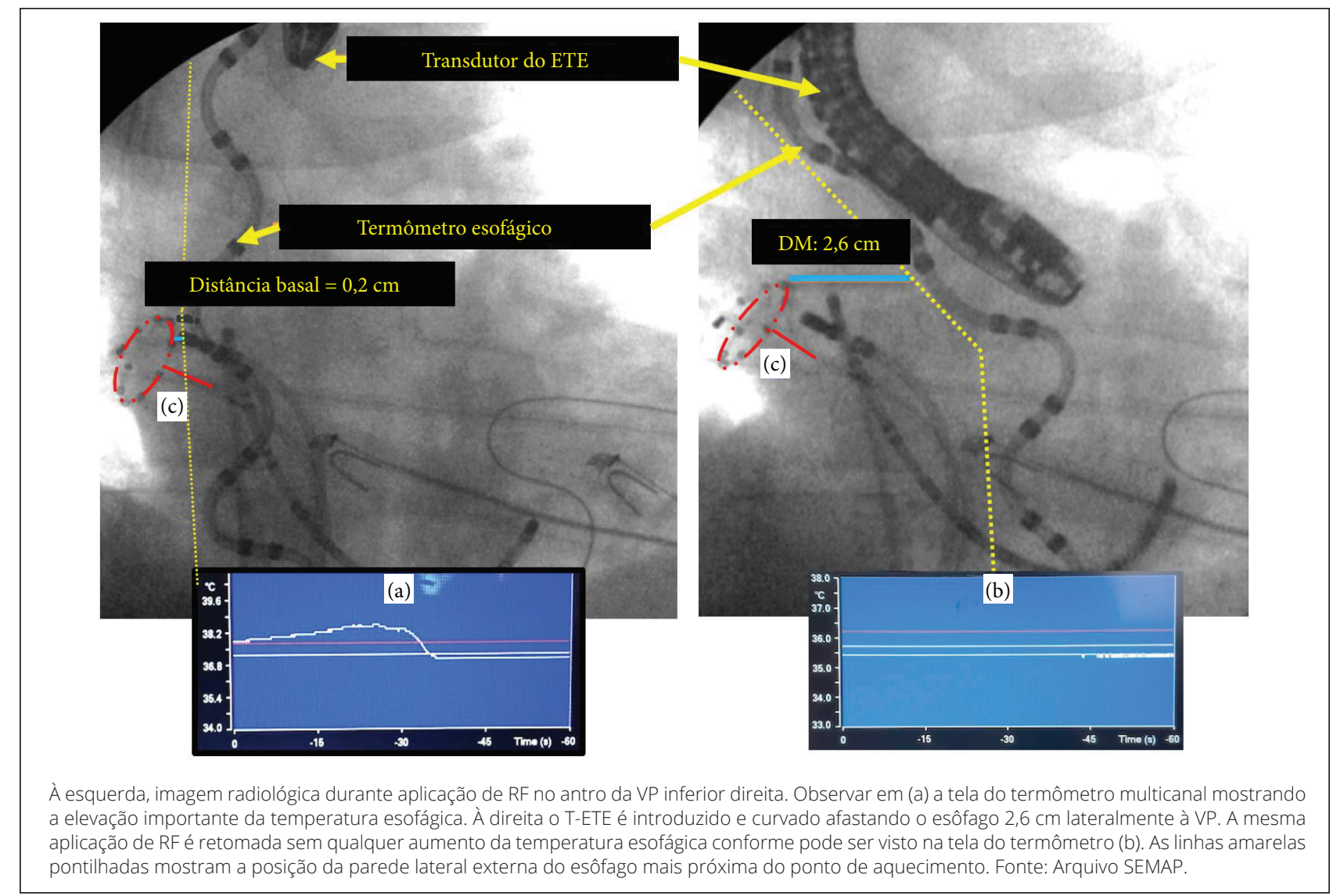

Figura 7. Eficiência do deslocamento do esôfago pela técnica de Pachón et al. ${ }^{53}$.

procedimento de ablação da FA é necessária para que essa técnica possa ser aplicada, o que pode ser considerado uma limitação dependendo do centro de eletrofisiologia. A mera presença do T-ETE no lúmen esofágico é considerada um fator de risco, uma vez que é capaz de trazer sua parede anterior mais próxima da parede posterior do $\mathrm{AE}$, onde as aplicações de RF são realizadas. Nessa técnica, é obrigatório realizar o desvio mecânico para um ponto contralateral, longe da aplicação da RF para garantir a segurança. De acordo com publicações anteriores de Pachón et al. ${ }^{53} \mathrm{e}$ Koruth et al. ${ }^{56}$ essa técnica não apresentou complicações importantes relacionadas à manipulação esofágica e nenhum registro de FAE.

Com relação a um possível efeito antena (por indução elétrica entre a ponta do cateter e o termômetro esofágico), isso não foi demonstrado com o uso de termômetros com isolamento elétrico dos sensores ${ }^{51}$. Aparentemente, esse efeito antena era produzido entre o equipamento que gera a $\mathrm{RF}$ e o equipamento que faz a medição da temperatura esofágica, desde que ambos não estivessem aterrados no mesmo ponto, produzindo uma diferença de potencial entre ambos e permitindo que a $\mathrm{RF}$ trafegasse também através dos polos do termômetro (como se fosse o eletrodo dispersivo) causando lesões nesses locais de contato. A partir da identificação desse problema, alguns fabricantes dos termômetros passaram a isolar eletricamente os sensores térmicos e também acrescentaram um aterramento auxiliar para igualar o potencial entre os dois equipamentos, eliminando esse problema. Quanto ao T-ETE, esse dispositivo é completamente isolado eletricamente, sendo que não há nenhum elemento metálico exposto (em condições normais) que possa produzir o mesmo efeito antena relatado para os termômetros. $\mathrm{O}$ T-ETE também possui um termômetro instalado na ponta, utilizado para monitorar a temperatura do cristal que produz o ultrassom e para evitar que seu aquecimento possa lesar a mucosa esofágica. Quando esse cristal atinge a temperatura de $38^{\circ} \mathrm{C}$, o equipamento desliga automaticamente o T-ETE para garantir a segurança do paciente. Esse termômetro não é adequado para monitorar a temperatura do esôfago durante a aplicação de RF.É importante salientar que, para desviar o esôfago, não é necessário que esse transdutor esteja ligado, produzindo qualquer aquecimento. 


\section{CONCLUSÃO}

O tratamento da fibrilação atrial por meio da radiofrequência vem apresentando grande desenvolvimento desde que foi iniciada em 1998 por Haïssaguerre. As complicações que surgiram devido ao procedimento, foram cada uma delas sendo corrigidas por meio da modificação das técnicas e dos equipamentos utilizados. A mais temida complicação é a fístula atrioesofágica, que, a despeito da sua baixa incidência, leva à morte a quase totalidade dos pacientes acometidos, apesar do tratamento instituído tão logo seja identificada. Em decorrência da elevada mortalidade, vários autores desenvolveram métodos para proteger o esôfago e reduzir essa complicação.

Os autores deste trabalho desenvolveram um método simples de deslocamento mecânico do esôfago, utilizando a sonda do ecocardiograma transesofágico. Esse transdutor é utilizado para avaliar a presença de trombos no início do procedimento, avaliar a presença de forame oval, auxiliar na punção transeptal, acompanhar todo o procedimento monitorando a presença de derrame pericárdico e também para deslocar o esôfago no momento da aplicação de radiofrequência no antro de cada veia pulmonar, fazendo com que o equipamento seja extremamente útil e seguro em todas as etapas da ablação da fibrilação atrial.

Mais de 3.000 procedimentos já foram realizados com esse método sem que tenha ocorrido uma única fístula atrioesofágica, comprovando que o deslocamento mecânico do esôfago é uma forma altamente simples, barata e segura de evitar essa grave complicação. Alguns fabricantes, apresentaram soluções para realizar o desvio do esôfago, que estão disponíveis para os serviços que não utilizam o ecocardiograma transesofágico rotineiramente durante o procedimento.

\section{REFERÊNCIAS}

1. Haïssaguerre $M$, Jaïs $P$, Shah $D C$, Takahashi $A$, Hocini $M$, Quiniou G, et al. Spontaneous initiation of atrial fibrillation by ectopic beats originating in the pulmonary veins. N Engl J Med, 1998;339(10):659-66. https://doi.org/10.1056/ NEJM199809033391003

2. Gerstenfeld EP, Guerra P, Sparks PB, Hattori K, Lesh MD. Clinical outcome after radiofrequency catheter ablation of focal atrial fibrillation triggers. J Cardiovasc Electrophysiol, 2001;12(8):9008. https://doi.org/10.1046/j.1540-8167.2001.00900.x

3. HaïssaguerreM,ShahDC, JaïsP,Hocini M, YamaneT, Deisenhofer I, et al. Electrophysiological breakthroughs from the left atrium to the pulmonary veins. Circulation, 2000;102(20):2463-5. https://doi.org/10.1161/01.cir.102.20.2463

4. Noheria A, Kumar A, Wylie Jr JV, Josephson ME. Catheter ablation vs antiarrhythmic drug therapy for atrial fibrillation: a systematic review. Arch Int Med 2008;168(6):581-6. https://doi.org/10.1001/archinte.168.6.581

5. Nielsen JC, Johannessen A, Raatikainen P, Hindricks G, Walfridsson $\mathrm{H}$, Kongstad $\mathrm{O}$, et al. Radiofrequency ablation as initial therapy in paroxysmal atrial fibrillation. N Engl J Med, 2012;367:1587-95. https://doi.org/10.1056/NEJMoa1113566

6. Li WJ, Bai YY, Zhang HY, Tang RB, Miao CL, Sang $\mathrm{CH}$ et al. Additional ablation of complex fractionated atrial electrograms after pulmonary vein isolation in patients with atrial fibrillation: a meta-analysis. Circ Arrhythm Electrophysiol, 2011;4(2):143-8.

7. Pachon JCM, EIM Pachon, JCM Pachon, TJ Lobo, MZC Pachon, Vargas RNA, et al. "AF Nests" electrical resonance.
Is it a new atrial fibrillation physiopathology? Heart Rhythm. 2005;2(5):S149. https://doi.org/10.1016/j.hrthm.2005.02.467

8. Mateos JCP, Mateos EIP, Lobo TJ, Pachón MZC, Mateos JCP, Pachón DQV, et al. Ablação da fibrilação atrial por cateter com radiofrequência guiada por mapeamento espectral endocárdico dos "ninhos de FA" em ritmo sinusal. Arq Bras Cardiol, 2007;89(3):140-50. https://doi.org/10.1590/ S0066-782X2007001500001

9. Mateos JCP, Mateos EIP, Santillana TGP, Lobo TJ, Pachón CTC, Mateos JCP, et al. Ablation of "background tachycardia" in long standing atrial fibrillation: improving the outcomes by unmasking a residual atrial fibrillation perpetuator. J Atr Fibrillation, 2017;10(2): 1583.

10. KnechtS, Hocini M, Wright M, Lellouche N, O'Neill MD, Matsuo S, et al. Left atrial linear lesions are required for successful treatment of persistent atrial fibrillation. Eur Heart J, 2008;29(19):2359-66. https://doi.org/10.1093/eurheartj/ehn302

11. Marrouche NF, Dresing T, Cole C, Bash D, Saad E, Balaban $\mathrm{K}$, et al. Circular mapping and ablation of the pulmonary vein for treatment of atrial fibrillation: impact of different catheter technologies. J Am Coll Cardiol, 2002;40(3):464-74. https://doi.org/10.1016/s0735-1097(02)01972-1

12. Schmidt M, Nölker G, Marschang $H$, Gutleben KJ, Schibgilla $V$, Rittger $H$, et al. Incidence of oesophageal wall injury postpulmonary vein antrum isolation for treatment of patients with atrial fibrillation. Europace 2008, 10(2):205-9. https:// doi.org/10.1093/europace/eun001 
13. Chavez P, Messerli FH, Casso Dominguez A, Aziz EF, Sichrovsky T, Garcia D, et al. Atrioesophageal fistula following ablation procedures for atrial fibrillation: systematic review of case reports. Open Heart, 2015;2(1):e000257. https:// doi.org/10.1136/openhrt-2015-000257

14. Kottkamp H, Hindricks G, Autschbach R, Krauss B, Strasser $B$, Schirdewahn P, et al. Specific linear left atrial lesions in atrial fibrillation: Intraoperative radiofrequency ablation using minimally invasive surgical techniques. J Am Coll Cardiol, 2002;40(3):475-80. https://doi.org/10.1016/S07351097(02)01993-9

15. Yousuf T, Keshmiri H, Bulwa Z, Kramer J, Arshad HMS, Issa $\mathrm{R}$, et al. Management of atrio-esophageal fistula following left atrial ablation. Cardiol Res. 2016;7(1):36-45. https://doi. org/10.14740/cr454e

16. Calkins H, Kuck KH, Cappato R, Brugada J, Camm AJ, Chen SA, et al. 2012 HRS/EHRA/ECAS expert consensus statement on catheter and surgical ablation of atrial fibrillation: recommendations for patient selection, procedural techniques, patient management and followup, definitions, endpoints, and research trial design. J Interv Card Electrophysiol, 33, 171-257 (2012). https://doi. org/10.1007/s10840-012-9672-7

17. Vasconcelos JTM, Galvão Filho SS, Atié J, Maciel W, Souza OF, Saad EB, et al. Atrial-oesophageal fistula following percutaneous radiofrequency catheter ablation of atrial fibrillation: the risk still persists. EP Europace, 2017;19(2):2508. https://doi.org/10.1093/europace/euw284

18. Kapur S, Barbhaiya C, Deneke T, Michaud GF. Esophageal injury and atrioesophageal fistula caused by ablation for atrial fibrillation. Circulation, 2017;136(13):1247-55. https:// doi.org/10.1161/CIRCULATIONAHA.117.025827

19. Cappato R, Calkins H, Chen SA, Davies W, lesaka Y, Kalman J, et al. Prevalence and causes of fatal outcome in catheter ablation of atrial fibrillation. J Am Coll Cardiol, 2009;53(19):1798-803. https://doi.org/10.1016/j.jacc.2009.02.022

20. Nair GM, Nery PB, Redpath CJ, Lam BK, Birnie DH. Atrioesophageal fistula in the era of atrial fibrillation ablation: a review. Can J Cardiol, 2014;30(4):388-95. https:// doi.org/10.1016/j.cjca.2013.12.012

21. Barbhaiya CR, Kumar S, Guo Y, Zhong J, John RM, Tedrow UB. Global survey of esophageal injury in atrial fibrillation ablation: characteristics and outcomes of esophageal perforation and fistula. JACC Clin Electrophysiol. 2016;2(2):143-50. https:// doi.org/10.1016/j.jacep.2015.10.013

22. Vijayaraman P, Netrebko P, Geyfman V, Dandamudi G, Casey K, Ellenbogen KA. Esophageal fistula formation despite esophageal monitoring and low-power radiofrequency catheter ablation for atrial fibrillation. Circ Arrhythmia Electrophysiol, 2009;2(5):e31-3. https://doi.org/10.1161/CIRCEP.109.883694

23. Jang SW, Kwon BJ, Choi MS, Kim DB, Shin WS, Cho EJ et al. Computed tomographic analysis of the esophagus, left atrium, and pulmonary veins: implications for catheter ablation of atrial fibrillation. J Interv Card Electrophysiol, 2011;32(1):1-6. https://doi.org/10.1007/s10840-011-9594-9
24. Maeda S, lesaka Y, Uno K, Otomo K, Nagata Y, Suzuki K, et al. Complex anatomy surrounding the left atrial posterior wall: analysis with 3D computed tomography. Heart Vessels, 2012;27(1):58-64. https://doi.org/10.1007/s00380-011-0120-x

25. Cury RC, Abbara S, Schmidt S, Malchano ZJ, Neuzil P, Weichet $J$, et al. Relationship of the esophagus and aorta to the left atrium and pulmonary veins: Implications for catheter ablation of atrial fibrillation. Heart Rhythm 2005 Dec;2(12):1317-23. https://doi.org/10.1016/j.hrthm.2005.09.012

26. Ueno T, Uemura K, Harris MB, Pappas TN, Takahashi T. Role of vagus nerve in postprandial antropyloric coordination in conscious dogs. Am J Physiol Gastrointest Liver Physiol. 2005;288(3):G487-95. https://doi.org/10.1152/ ajpgi.00195.2004

27. Nair KKM, Danon A, Valaparambil A, Koruth JS, Singh SM. Atrioesophageal fistula: a review J Atr Fibrillation. 2015 OctNov; 8(3):1331.

28. Martinek M, Bencsik $G$, Aichinger J, Hassanein S, Schoefl R, Kuchinka P, et al. Esophageal damage during radiofrequency ablation of atrial fibrillation: impact of energy settings, lesion sets, and esophageal visualization. J Cardiovasc Electrophysiol, 2009 Jul;20(7):726-33. https:// doi.org/10.1111/j.1540-8167.2008.01426.x

29. Calzolari V, Mattia L, Indiani S, Crosato M, Furlanetto A, Licciardello $C$, et al. In vitro validation of the lesion size index to predict lesion width and depth after irrigated radiofrequency ablation in a porcine model. JACC Clin Electrophysiol, 2017;3(10):1126-35. https://doi. org/10.1016/j.jacep.2017.08.016

30. Chavez P, Messerli FH, Casso Dominguez A, Aziz EF, Sichrovsky T, Garcia D, et al. Atrioesophageal fistula following ablation procedures for atrial fibrillation: systematic review of case reports. Open Heart, 2015;2(1):e000257. https:// doi.org/10.1136/openhrt-2015-000257

31. Halbfass P, Pavlov B, Müller $P$, Nentwich $K$, Sonne $K$, Barth $S$, et al. Progression from esophageal thermal asymptomatic lesion to perforation complicating atrial fibrillation ablation: a single-center registry. Circ Arrhythm Electrophysiol, 2017;10(8):e005233. https://doi.org/10.1161/ CIRCEP.117.005233

32. Han HC, Ha FJ, Sanders P, Spencer R, Teh AW, O'Donnell D, et al. Atrioesophageal fistula clinical presentation, procedural characteristics, diagnostic investigations, and treatment outcomes. Circ Arrhythm Electrophysiol, 2017;10:e005579. https://doi.org/10.1161/CIRCEP.117.005579

33. Linz D, Hohl M, Vollmar J, Ukena C, Mahfoud F, Böhm M. Atrial fibrillation and gastroesophageal reflux disease: the cardiogastric interaction. Europace. 2017;19(1):16-20. https://doi.org/10.1093/europace/euw092

34. Garg L, Garg J, Gupta N, Shah N, Krishnamoorthy P, Palaniswamy C, et al. Gastrointestinal complications associated with catheter ablation for atrial fibrillation. Int J Cardiol, 2016;224:424-30. https://doi.org/10.1016/j.ijcard.2016.09.069 
35. Vasconcelos JTM, Galvão Filho SS, Atié J, Maciel W, Souza OF, Saad EB, et al. Atrial-oesophageal fistula following percutaneous radiofrequency catheter ablation of atrial fibrillation: the risk still persists. Europace. 2017;19(2):25058. https://doi.org/10.1093/europace/euw284

36. January CT, Wann LS, AlpertJS, Calkins H, CigarroaJE, Cleveland Jr JC, et al. 2014 AHA/ACC/HRS guideline for the management of patients with atrial fibrillation: executive summary: a report of the American College of Cardiology/American Heart Association Task Force on practice guidelines and the Heart Rhythm Society.. Circulation. 2014;130(23):2071-104. https:// doi.org/10.1161/CIR.0000000000000040

37. Scanavacca M. Ablação de FA e risco de lesão esofágica. Arq Bras Cardiol, 2016;106(5):354-7.

38. Singh SM, d'Avila A, Singh SK, Stelzer P, Saad EB, Skanes $A$, et al. Clinical outcomes after repair of left atrial esophageal fistulas occurring after atrial fibrillation ablation procedures. Heart Rhythm, 2013;10(11):1591-7. https:// doi.org/10.1016/j.hrthm.2013.08.012

39. Stárek Z, Lehar F, Jež J, Žbánková A, Kulík T, Wolf J, Novák M. Long-term mobility of the esophagus in patients undergoing catheter ablation of atrial fibrillation: data from computer tomography and 3D rotational angiography of the left atrium. J Interv Card Electrophysiol, 2016; 46(2):81-7. https://doi.org/10.1007/s10840-016-0121-x

40. Daoud EG, Hummel JD, Houmsse M, Hart DT, Weiss R, Liu Z, et al. Comparison of computed tomography imaging with intraprocedural contrast esophagram: implications for catheter ablation of atrial fibrillation. Heart Rhythm. 2008;5(7):975-80. https://doi.org/10.1016/j.hrthm.2008.03.058

41. Good E, Oral H, Lemola K, Han J, Tamirisa K, Igic P, et al. Movement of the esophagus during left atrial catheter ablation for atrial fibrillation. J Am Coll Cardiol, 2005;46(11):2107-10. https://doi.org/10.1016/j.jacc.2005.08.042

42. Bunch TJ, May HT, Crandall BG, Weiss JP, Bair TL, Osborn JS, et al. Intracardiac ultrasound for esophageal anatomic assessment and localization during left atrial ablation for atrial fibrillation. J Cardiovasc Electrophysiol, 2013;24(1):339. https://doi.org/10.1111/j.1540-8167.2012.02441.x

43. Bahnson TD. Strategies to minimize the risk of esophageal injury during catheter ablation for atrial fibrillation. Pacing Clin Electrophysiol, 2009;32(2):248-60. https://doi. org/10.1111/j.1540-8159.2008.02210.x

44. Lemola K, Sneider M, Desjardins B, Case I, Han J, Good E, et al. Computed tomographic analysis of the anatomy of the left atrium and the esophagus: implications for left atrial catheter ablation. Circulation, 2004;110(24):3655-60. https://doi.org/10.1161/01.CIR.0000149714.31471.FD

45. Contreras-Valdes FM, Heist EK, Danik SB, Barrett CD, Blendea $D$, Brugge WR, et al. Severity of esophageal injury predicts time to healing after radiofrequency catheter ablation for atrial fibrillation. Heart Rhythm, 2011;8(12):1862-6. https:// doi.org/10.1016/j.hrthm.2011.07.022

46. Di Biase L, Dodig M, Saliba W, Siu A, Santisi J, Poe S, et al. Capsule endoscopy in examination of esophagus for lesions after radiofrequency catheter ablation: a potential tool to select patients with increased risk of complications. J Cardiovasc Electrophysiol, 2010;21(8):839-84. https://doi. org/10.1111/j.1540-8167.2010.01732.x

47. Halm U, Gaspar T, Zachäus M, Sack S, Arya A, Piorkowski C, et al. Thermal esophageal lesions after radiofrequency catheter ablation of left atrial arrhythmias. Am J Gastroenterol, 2010;105(3):551-6. https://doi.org/10.1038/ajg.2009.625

48. Singh SM, d'Avila A, Doshi SK, Brugge WR, Bedford RA, Mela T, et al. Esophageal injury and temperature monitoring during atrial fibrillation ablation. Circ Arrhythm Electrophysiol, 2008;1(3):162-8. https://doi.org/10.1161/ CIRCEP.107.789552

49. Müller P, Dietrich JW, Halbfass P, Abouarab A, Fochler F, Szöllösi A, et al. Higher incidence of esophageal lesions after ablation of atrial fibrillation related to the use of esophageal temperature probes. Heart Rhythm, 2015;12(17):1464-9. https://doi.org/10.1016/j.hrthm.2015.04.005

50. Halbfass P, Müller P, Nentwich K, Krug J, Roos M, Hamm K, et al. Incidence of asymptomatic oesophageal lesions after atrial fibrillation ablation using an oesophageal temperature probe with insulated thermocouples: a comparative controlled study. Europace, 2017;19(3):385-91. https://doi. org/10.1093/europace/euw070

51. Kuwahara T, Takahashi A, Okubo K, Takagi K, Yamao K, Nakashima E, et al. Oesophageal cooling with ice water does not reduce the incidence of oesophageal lesions complicating catheter ablation of atrial fibrillation: randomized controlled study. Europace, 2014;16(6):834-9. https://doi.org/10.1093/europace/eut368

52. Arruda MS, Armaganijan L, Di Biase L, Rashidi R, Natale A. Pre clinical "in vivo" evaluation of an esophageal protective system: implications on esophageal thermal injury during AF ablation. Heart Rhythm, 2008;5:S16. https://doi. org/10.1016/j.hrthm.2008.03.040

53. Pachón JCM, Mateos El, Peña TG, Lobo TJ, Mateos JC, Vargas RN. Simplified method for esophagus protection during radiofrequency catheter ablation of atrial fibrillation - prospective study of 704 cases. Rev Bras Cir Cardiovasc, 2015;30(2):139-47. https://doi.org/10.5935/1678-9741.20150009

54. Amarante RC. Eficácia e segurança da técnica do desvio mecânico na prevenção do aquecimento do esôfago na ablação da fibrilação atrial com cateter por radiofrequência. São Paulo. Tese [Doutorado em Ciências] - Universidade de São Paulo; 2019.

55. Chugh A, Rubenstein J, Good E, Ebinger M, Jongnarangsin K, Fortino J, et al. Mechanical displacement of the esophagus in patients undergoing left atrial ablation of atrial fibrillation. Heart Rhythm, 2009;6(3):319-22. https://doi.org/10.1016/j. hrthm.2008.12.010

56. KoruthJS, Reddy VY, Miller MA, Patel KK, Coffey JO, Fischer A, et al. Mechanical esophageal displacement during catheter ablation for atrial fibrillation. J Cardiovasc Electrophysiol, 2012;23(2):14754. https://doi.org/10.1111/j.1540-8167.2011.02162.x 